\title{
Triangular Rogue Waves and Multi- Wave Trains Generation in a Chameleon Electrical Transmission Line
}

\author{
Bedel Giscard Onana Essama ${ }^{1,}$, , Salome Ndjakomo Essiane ${ }^{1}$, Frederic Biya - Motto ${ }^{2}$, \\ Mohammed Shabat ${ }^{3}$, Jacques Atangana ${ }^{4}$ \\ ${ }^{1}$ Higher Technical Teachers Training College (HTTTC) of Ebolowa, University of Yaoundé I, Ebolowa, Cameroon \\ ${ }^{2}$ Department of Physics, Faculty of Science, University of Yaounde I, Yaounde, Cameroon \\ ${ }^{3}$ Department of Physics, Islamic University of Gaza, Gaza City, Palestine \\ ${ }^{4}$ Higher Teacher Training College of Yaoundé, University of Yaoundé I, Yaoundé, Cameroon
}

Email address:

onanaessama@yahoo.fr (B. G. O. Essama)

${ }^{*}$ Corresponding author

\section{To cite this article:}

Bedel Giscard Onana Essama, Salome Ndjakomo Essiane, Frederic Biya - Motto, Mohammed Shabat, Jacques Atangana. Triangular Rogue Waves and Multi- Wave Trains Generation in a Chameleon Electrical Transmission Line. American Journal of Optics and Photonics. Vol. 8, No. 3, 2020, pp. 61-73. doi: 10.11648/j.ajop.20200803.12

Received: August 9, 2020; Accepted: August 26, 2020; Published: November 23, 2020

\begin{abstract}
In this work, we present a particular nonlinear transmission line called chameleon nonlinear transmission line. In fact, the chameleon's behavior is related to the fact that without changing its appearance, the line can exhibit alternatively purely right - or left - handed behavior. This transmission line is different to the composite one. So, the goal of this work is to demonstrate that this special line can support the propagation of soliton light pulse subjected to quintic - phase modulation, improve the freak wave's mechanism of generation and verify the chameleon's behavior of the line. Consequently, we employ collective coordinate's theory in order to give a great characterization of the light pulse. Then, we introduce an upgraded function called "type II Ansatz function" with eight collective coordinates compared to the conventional Gaussian Ansatz with six collective coordinates. We show that the two additional coordinates will allow us to improve the technique of measurement of internal excitation leading to the generation of rogue events. Moreover, these coordinates will give supplemental details on frequency shift and chirp distortions during the generation of specific rogue events such as "wall of waves", tree structures, multi - wave trains, Kuznetsov - Ma breathers, Akhmediev breathers, Peregrine solitons and triangular rogue waves. The stability of the soliton light pulse will be also investigated at specific frequency ranges.
\end{abstract}

Keywords: Triangular Rogue Waves, Chameleon Transmission Line, Multi - Wave Trains, Tree Structures, Collective Coordinates

\section{Introduction}

Metamaterials are materials which present a negative refraction index compared to ordinary materials with positive one $[1,2]$. Consequently, this type of materials which is not found in nature is named negative index, double - negative or left - handed metamaterials [1, 2]. Further, transmission lines are found at microwave frequencies zone [3, 4]. This discovery leads to left - handed transmission line capable to support soliton light pulses propagation [5 - 11]. Thereafter, composite right/left - handed transmission lines are also found where left - handed behavior is presented at low frequencies and right - handed behavior at high frequencies [12 - 18].

Recently, a new field of investigation has emerged where extreme events lead to many physical applications [19 - 21]. Furthermore, extreme events are found in several domains such as nonlinear optics [22], lasers [23], and plasmas [24]. In addition, some interesting extreme events have been studied such as Peregrine solitons [25], Kuznetsov - Ma breathers [26 - 28], Akhmediev breathers [26, 29 - 31] in the nonlinear Schrödinger equation [32]. The most important freak wave's mechanism of generation is modulation instability which fundamentally acts on nonlinear dispersive systems [33]. In optics, modulation instability originates 
from noise results in a series of high - contrast peaks of random intensity $[34,35]$. Besides, it is these localized peaks that have been compared with similar structures seen in studies of ocean rogue waves [32, 36, 37]. Modulation instability has been intensively studied in many domains such as nonlinear optics [38], plasma physics [39], hydrodynamics [40], electrical transmission lines [41], Bose-Einstein condensates [42 - 44], and optical fibers [45].

However, a strange phenomenon called "tree structure" which is directly related to chaotic waves field due to modulation instability, has been recently outlined [46]. Some studies have been made concerning the so - called "tree structure". Among them, we have the "Christmas tree" which appears during the formation of Peregrine waves [47]. Such phenomenology has been firstly observed and extensively analyzed in [48], which is linked to the umbilical gradient catastrophe. Moreover, Raman Effect can induce the appearance of particular "tree structure" with roots which can be called "roots of propagation" [46, 49]. Others authors such as Dudley [50], sustains that such "tree structures" are corresponded to signatures of analytic nonlinear Schrödinger equation solutions in chaotic modulation instability. Further, several investigations in metamaterials including extreme events have been presented in literature [47, 51 - 54], but some others have employed collective coordinates theory [ 46 55 - 60]. To the best of our knowledge, the use of an upgraded function called "type II ansatz function" [61], in order to give an exact measure of internal excitation leading to the generation of special extreme events such as "wall of waves", tree structures, triangular rogue waves and multiwave trains, has been least reported in literature.

In this paper, according to considerations made in $[41,62$, 63], we use an upgraded function called "type II Ansatz function" with eight coordinates [61], compared to the conventional Gaussian Ansatz with six coordinates [46, 55 60]. The so - called "type II Ansatz function" introduces two additional coordinates which give supplemental details on internal agitation leading to freak wave's generation. We introduce at the first time quintic - phase modulation on a specific line called chameleon transmission line. So, the quintic - phase modulation strength of distortion is strongly evaluated in order to improve the comprehension of freak wave's mechanism of generation. Some interesting freak events due to quintic - phase modulation have been found such as the so - called "wall of waves", tree structures, multi - wave trains, triangular freak waves, Peregrine solitons [64, 65], Akhmediev [66 - 68] and Kuznetsov - Ma breathers. Further, the internal agitation which modifies the system in a special way when each of the above mentioned freak events comes into play, is well measured. The chameleon's behavior of this particular line is specially verified. We organize the paper as follows. In Section 2, we present the model of chameleon transmission line and give some equations such as logarithmic nonlinearity for the capacitance, the voltage propagation equations and the nonlinear Schrödinger equation model. The coefficients of this last equation are calculated and linked to frequency and dimensionless capacitor. Thereafter, we apply the collective coordinate's technique [69 - 73], employing the so - called "type II AnsatzS function" with eight coordinates [61]. Two additional coordinates are introduced respectively called frequency shift correction factor and chirp correction factor [61]. These two coordinates will give supplemental details on internal distortions which occur during the freak wave's generation. Furthermore, we obtain collective coordinate equations of motion. In Section 3, we present computational results. Some interesting results including the verification of chameleon's behavior of the line, the exact measure of internal and external excitation leading to the generation of freaks waves such as the so - called "wall of waves", tree structures, multi - wave trains, triangular freak waves, Peregrine solitons, Akhmediev and Kuznetsov - Ma breathers, are presented. Otherwise, specific frequency ranges where the soliton regains its stability are also presented. Indeed, the special influence of dimensionless capacitor and that of frequency on freak wave's mechanism of generation are also investigated. We compare the results obtained with previous analytical and numerical investigations. We summarize the investigation in Section 4.

\section{Mathematical Description of the Model}

\subsection{Preliminaries}

The model of line under consideration represents a nonlinear electrical transmission line where elementary cell is illustrated in Figure $1[62,63]$.

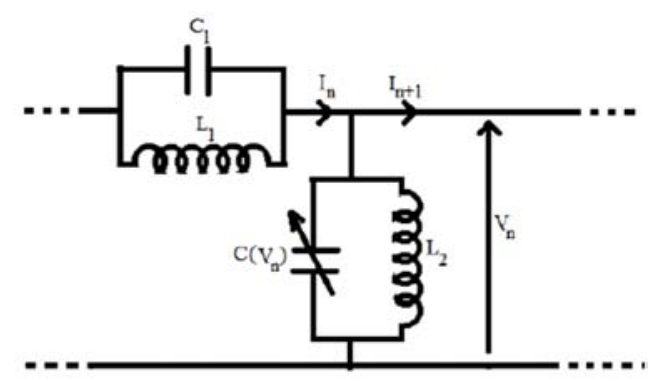

Figure 1. Model for the unit-cell circuit.

Each unit cell, such as the $n^{\text {th }}$ one, contains a linear inductor $L 1$ in parallel with a linear capacitor $C 1$ in the series branch and a linear inductor $L 2$ in parallel with a nonlinear capacitor $C(V n)$ in the shunt branch. Here we assume that the logarithmic nonlinearity for the capacitance is given by $[74,75]$ :

$$
C\left(V_{n}\right)=\frac{V_{0} C_{0}}{V_{n}} \ln \left(1+\frac{V_{n}}{V_{0}}\right)
$$

where $V 0$ and $C 0$ take constant values. Applying Kirchhoff's laws to the circuit model, we can obtain the following voltage propagation equation [63]:

$$
\begin{gathered}
\left(V_{0}+V_{n}\right) \frac{d^{2} V_{n}}{d t^{2}}-\left(\frac{d V_{n}}{d t}\right)^{2}=\frac{\left(V_{0}+V_{n}\right)^{2}}{V_{0}}\left\{( u _ { 0 } ^ { 2 } + C _ { r } \frac { d ^ { 2 } } { d t ^ { 2 } } ) \left(V_{n+1}+\right.\right. \\
\left.\left.V_{n-1}-2 V_{n}\right)-\omega_{0}^{2} V_{n}\right\}
\end{gathered}
$$


where $C_{r}=\frac{C_{1}}{C_{0}}, u_{0}^{2}=\frac{1}{L_{1} C_{0}}, \omega_{0}^{2}=\frac{1}{L_{2} C_{0}}$. Equation (2) shows that an additional dispersion coefficient $\mathrm{Cr}$ is considered on the line. Thereafter, the numerical simulations will consider the following parameters [41, 63]: $L 1=680 \mu H, L 2=470 \mu H, C 0$ $=470 \mathrm{pF}$ and $V 0=3.9 \mathrm{~V}$. The capacitor $C 1$ is a free parameter with physically acceptance value [63]. Moreover, the quantity $\mathrm{Cr}$ significantly modifies the behavior of the transmission line [63].

Considering $\mathrm{Cr}=0[41,63]$, the line adopts the right handed behavior, but for $C_{r}>\frac{u_{0}^{2}}{\omega_{0}^{2}}=\frac{L_{2}}{L_{1}}$, the transmission line exhibits left - handed behavior. It is important to note that a rapid calculation leads to $\frac{L_{2}}{L_{1}} \approx 0.69$.

\subsection{The Model}

We establish the nonlinear Schrödinger equation according to the considerations made in [63], but reformulated in terms of slowly varying envelope of the electric field $\varphi(Z, \tau)$ as follows [76]:

$$
\frac{\partial \varphi}{\partial Z}=-i \frac{\Delta_{2}}{2} \frac{\partial^{2} \varphi}{\partial \tau^{2}}+i \Gamma_{0}|\varphi|^{2} \varphi+i \Gamma_{a}|\varphi|^{4} \varphi .
$$

The quantities $Z=\varepsilon\left(n-V_{g} t\right)$ and $\tau=\varepsilon^{2} t$ stand for the position and the time, respectively [63]. Here $\varepsilon$ is a positive and small parameter. The terms $\Delta 2, \Gamma 0$ and $\Gamma a$ are second order dispersion, self - and quantic - phase modulations, respectively [55 - 58]. These above mentioned coefficients are defined as follows [63]:

$$
\begin{gathered}
\Delta_{2}=-\frac{V_{g}^{2}}{2 \omega}+\frac{\left(u_{0}^{2}-C_{r} \omega^{2}\right) \cos (k)-4 C_{r} V_{g} \omega \sin (k)}{2 \omega\left[1+4 C_{r} \sin ^{2}\left(\frac{k}{2}\right)\right]} \\
\Gamma_{0}=\frac{3 \omega}{2 V_{0}^{2}}\left[-1+\frac{8 C_{r} \sin ^{2}\left(\frac{k}{2}\right)}{1+4 C_{r} \sin ^{2}\left(\frac{k}{2}\right)}\right]
\end{gathered}
$$

The wave number $\mathrm{k}$ is taken in the Brillouin zone. This dispersion relation admits two cutoff frequencies at $k=0$ rad.Cell ${ }^{-1}$ and $k=\pi$ rad.Cell ${ }^{-1}$ [63]. The quantity, $V g$, is the group velocity given by [63]:

$$
V_{g}=\frac{\left(u_{0}^{2}-C_{r} \omega_{0}^{2}\right) \sin (k)}{\omega\left[1+4 C_{r} \sin \left(\frac{k}{2}\right)^{2}\right]^{2}}
$$

The term, $\Gamma a$, called quintic - phase modulation is directly linked to self - phase modulation such as [61]:

$$
\Gamma_{a}=\alpha \Gamma_{0}
$$

Where $\alpha$ stands for a constant parameter. The parameter, $\Gamma a$, is introduced at the first time in this particular line. During the investigation, we will evaluate the specific disturbance provoked by this effect on the line. The special properties of this particular line will probably influence the internal agitation due to quintic - phase modulation in order to build up extreme events.

\subsection{Collective Coordinate's Theory}

\subsubsection{Type II Ansatz Function}

The collective coordinate's technique is a great method of characterization of a light pulse intensity profile using Ansatz functions $[61,70,77]$. The minimization of the residual field is crucial to determine the collective coordinate equations of motion also called variational equations. These variational equations are essential to obtain a good description of the light pulse [55 - 58, 61, 69 - 73, 77].

Previous investigations have demonstrated that the Gaussian Ansatz function cannot reconstruct the chaotic behavior exhibited by rogue events [46, 55 - 58, 61]. However, same works have shown that conventional or Gaussian Ansatz function can present several internal details concerning the internal excitation leading to the generation of freak events. In this paper, we introduce an upgraded Ansatz function with eight collective coordinates [61]. This new function can be called "type II Ansatz function". Compared to Gaussian Ansatz function, type II Ansatz function introduces two additional collective coordinates in order to improve the comprehension of rogue wave's mechanism of generation.

The upgraded Ansatz function also called type II Ansatz function with eight collective coordinates is given as [61]:

$$
\begin{gathered}
F(Z, \tau)=X_{1}(Z) \exp \left[-\left(\frac{\tau-X_{2}(Z)}{X_{3}(Z)}\right)^{2}+i\left(\frac{X_{8}(Z)}{4}\right)\left(\tau-X_{2}(Z)\right)^{4}+i\left(\frac{X_{7}(Z)}{2}\right)\left(\tau-X_{2}(Z)\right)^{3}+i\left(\frac{X_{4}(Z)}{2}\right)\left(\tau-X_{2}(Z)\right)^{2}+\right. \\
\left.i X_{5}(Z)\left(\tau-X_{2}(Z)\right)+i X_{6}(Z)\right]
\end{gathered}
$$

The quantities $X_{1}, X_{2}, \sqrt{2 \log 2} X_{3}, \frac{X_{4}}{2 \pi}, X_{5}, X_{6}$ are the conventional collective coordinates often used to represent the pulse amplitude, temporal position, full width at halfmaximum (FWHM) of peak power, chirp, frequency shift and phase, respectively [55 - 59, 61, 70, 77].

We have two additional collective coordinates, $X 7$ and $X 8 / 2 \pi$, respectively called frequency shift correction factor and the chirp correction factor [61]. The coordinate $X 7$ reconstructs all additional frequency fluctuations able to give a good description of the soliton self - frequency shift behavior. However, the coordinate $X 8$ reconstructs a best chirp dynamics and complete the insufficiencies of the conventional chirp dynamics coming from the Gaussian Ansatz function [61]. Consequently, a good estimation of the frequency and temporal shifts depends on the level of precision given by type II Ansatz function.

According to previous works, as shown by conventional Ansatz function [55 - 59, 61, 70, 77], type II Ansatz function will not be able to reconstruct the chaotic behavior presented by rogue events. However, the two additional collective coordinates will probably give supplemental details on internal perturbation leading to the generation of extreme events.

\subsubsection{Collective Coordinate Equations of Motion}

Then, the equations of motion obtained from bare 
approximation $[58,61,70,77]$, are given by the following expressions [61, 69]:

$$
\begin{gathered}
\dot{X}_{1}(Z)=\left(\frac{1}{2} X_{1} X_{4}+\frac{3}{8} X_{1} X_{3}^{2} X_{8}\right) \Delta_{2}(Z) \\
\dot{X}_{2}(Z)=\left(-X_{5}-\frac{3}{8} X_{3}^{2} X_{7}\right) \Delta_{2}(Z) \\
\dot{X}_{3}(Z)=\left(-X_{3} X_{4}-\frac{3}{4} X_{3}^{3} X_{8}\right) \Delta_{2}(Z) \\
\dot{X}_{4}(Z)=\left(X_{4}^{2}-\frac{4}{X_{3}^{4}}-\frac{45}{16} X_{3}^{4} X_{8}^{2}-\frac{9}{8} X_{3}^{2} X_{7}^{2}\right) \Delta_{2}(Z)-\left(\frac{3 \sqrt{2}}{4} \frac{X_{1}^{2}}{X_{3}^{2}}+\sqrt{2} \frac{1}{X_{3}^{2}}\right) \Gamma_{0}(Z)-\frac{8 \sqrt{3}}{9} \frac{X_{1}^{4}}{X_{3}^{2}} \Gamma_{a}(Z) \\
\dot{X}_{5}(Z)=\left(-\frac{45}{32} X_{3}^{4} X_{7} X_{8}-\frac{3}{8} X_{3}^{2} X_{4} X_{7}\right) \Delta_{2}(Z) \\
\dot{X}_{6}(Z)=\left(\frac{1}{X_{3}^{2}}-\frac{1}{2} X_{5}^{2}+\frac{15}{128} X_{3}^{6} X_{8}^{2}-\frac{3}{8} X_{3}^{2} X_{5} X_{7}\right) \Delta_{2}(Z)+\frac{43 \sqrt{2}}{64} X_{1}^{2} \Gamma_{0}(Z)-\frac{41 \sqrt{3}}{72} X_{1}^{4} \Gamma_{a}(Z) \\
\dot{X}_{7}(Z)=\left(\frac{27}{4} X_{3}^{2} X_{7} X_{8}+3 X_{4} X_{7}\right) \Delta_{2}(Z) \\
\dot{X}_{8}(Z)=\left(\frac{15}{2} X_{3}^{2} X_{8}^{2}+4 X_{4} X_{8}+\frac{9}{2} X_{7}^{2}\right) \Delta_{2}(Z)+\sqrt{2} \frac{X_{1}^{2}}{X_{3}^{4}} \Gamma_{0}(Z)+\frac{32 \sqrt{3}}{27} \frac{X_{1}^{4}}{X_{3}^{4}} \Gamma_{a}(Z)
\end{gathered}
$$

Equations (9) - (16) present the variational equations which are function of the coefficients $\Delta 2(Z), \Gamma 0(Z)$ and $\Gamma a(Z)$. All these equations are influenced by $\Delta 2(Z)$. The parameter, $\Delta 2(Z)$, responsible to second -order dispersion, maintains a symmetric behavior of the pulse. Moreover, the dynamics of frequency shift and that of frequency shift correction factor are strongly influenced by second - order dispersion. The quantities, $\Gamma 0(Z)$ and $\Gamma a(Z)$ influence the dynamic of chirp, phase and chirp correction factor. So, quintic - phase modulation will probably introduce additional modifications on the phase and chirp during the generation of rogue events.

\section{Computational Results}

\subsection{Computational Conditions for Electrical Transmission Line}

The conditions at the beginning of the propagation are inspired to those mentioned in [55 - 58]. According to the analysis of our optical system the wave number is taken as $k=$ $3 \mathrm{rad} \mathrm{Cell}^{-1}$. Otherwise, two lengths of propagation will be taken such as $\mathrm{Z}=3 \times 10^{-24} \mathrm{~m}$ and $\mathrm{Z}=10^{-6} \mathrm{~m}$ in order to make a great evaluation of the strange behavior of the line. According to those distances the previous linear and nonlinear effects can act on electrical transmission line. Concerning the collective coordinate representation, the dotted red curves correspond to the dynamics of collective coordinates obtained from bare approximation (Type II Ansatz function) as depicted in Figure 2(a). Further, the solid black curves represent the dynamics of collective coordinates coming from minimization [46]. Moreover, the dashed green curve gives the residual field energy (RFE) [55 - 58], which outlines the level of internal agitation of the light pulse. In addition, at the field representations, we respectively have the dashed blue curve which stands for the exact field solution of nonlinear Schrödinger equation, the solid black curve which corresponds to the field reconstructed by collective coordinates coming from minimization and the dotted red curve is the field reconstructed by collective coordinates originating from type II Ansatz function. Figure 2(d) represents the full numerical equation [55 - 58]. Moreover, Figure 2(f) shows the 2D full numerical equation $[49,50,54,78-83]$.

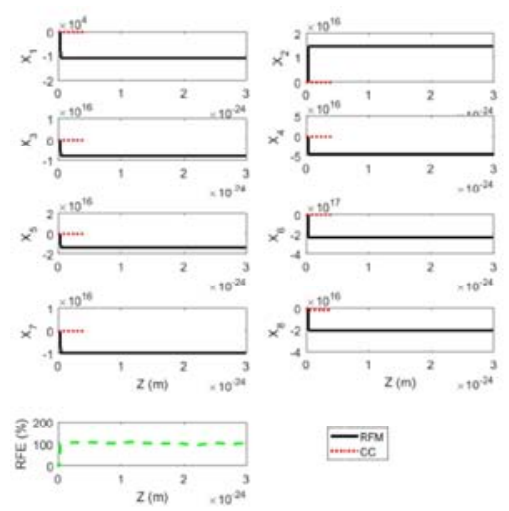

(a)

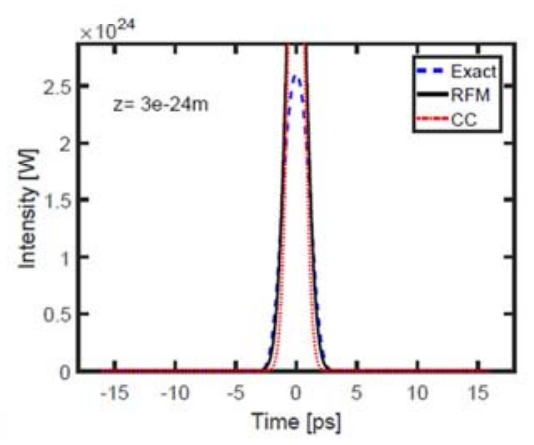




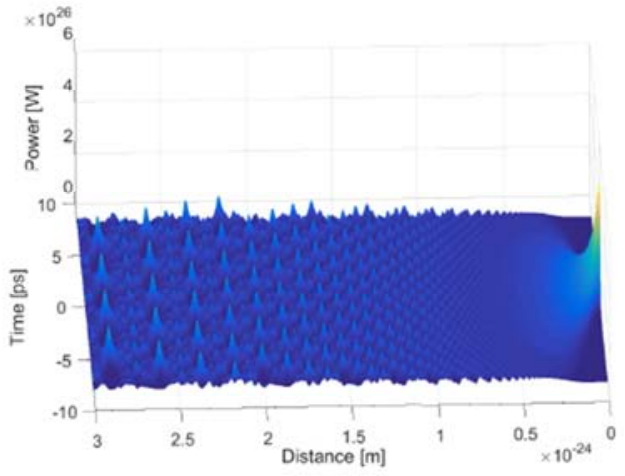

(c)

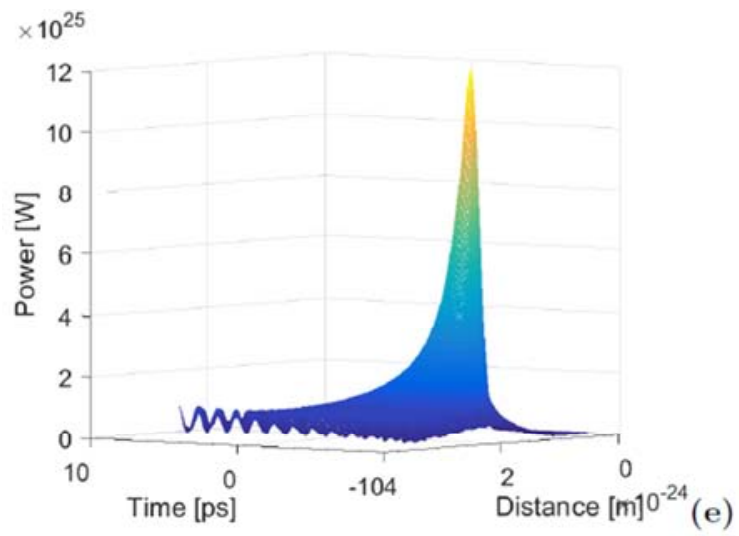

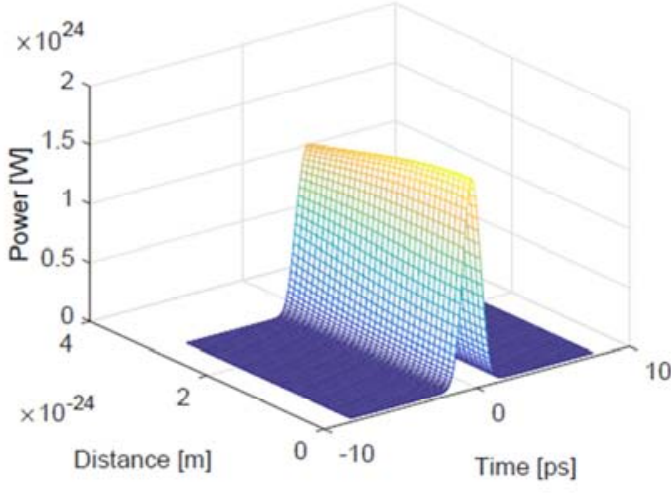

(d)

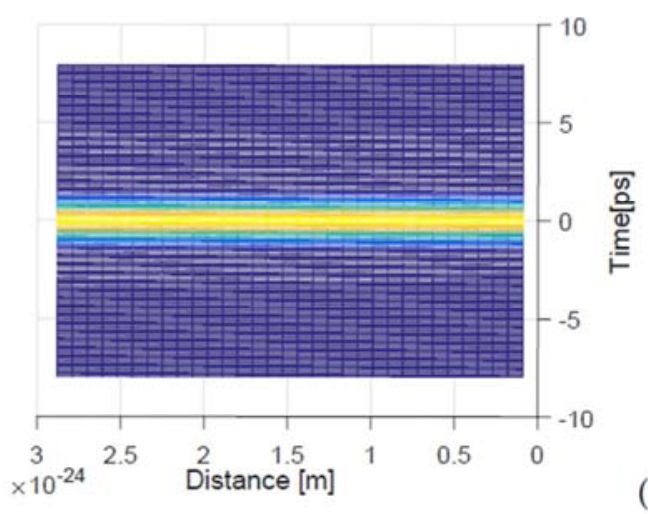

(f)

Figure 2. Generation of Akhmediev breathers and Peregrine soliton at $Z=3 \times 10^{-24} \mathrm{~m}$ for Cr=0 (Right - handed behavior). Soliton light pulse subjected to quintic - phase modulation $\alpha=0.2 \times 10^{-25}$ : (a) Dynamic of collective coordinates when $\omega=0.25 \mathrm{rad} / \mathrm{s}$; (b) Intensity of fields when $\omega=0.78 \mathrm{rad} / \mathrm{s}$; Full numerical equations: (c) $\omega=0.25 \mathrm{rad} / \mathrm{s}$, (d) $\omega=1 \mathrm{rad} / \mathrm{s}$ and (e) $\omega=0.35 \mathrm{rad} / \mathrm{s} ;$ (f) $2 \mathrm{D}$ full numerical equation when $\omega=5 \mathrm{rad} / \mathrm{s}[\mathrm{Frequency} \mathrm{ranges} \mathrm{considered:} 0<\omega \leq 0.78$ $\mathrm{rad} / \mathrm{s}$ and $\omega>0.78 \mathrm{rad} / \mathrm{s}]$.

\subsection{Right - Handed Behavior of Electrical Transmission Line}

The right - handed behavior occurs on the line when $0 \leq C_{r}<0.69$ [63]. Consequently, two cases will be investigated known as $\mathrm{Cr}=0$ and $\mathrm{Cr}=0.3$.

\subsubsection{Akhmediev Breathers and Peregrine Solitons}

We consider the first case of right - handed behavior such as $C r=0$ [41], at the distance $\mathrm{Z}=3 \times 10^{-24} \mathrm{~m}$. We study the behavior of the light pulse at low frequencies range such as 0 $<\omega<0.78 \mathrm{rad} / \mathrm{s}$. Considering the frequency as $\omega=0.25$ $\mathrm{rad} / \mathrm{s}$, second - order dispersion, self - and quintic - phase modulations come into play. So, the combination of those effects induces the appearance of Figure 2. Besides, Figure 2(c) is the well-known Akhmediev - Peregrine rogue wave's field. This wave's field is similar to that previously investigated in [60]. The field depicted in [60], has been generated by the interaction between second - order dispersion and cubic - nonlinearity in the composite right/left -handed transmission line. However, the wave's field illustrated in Figure 2(c) is induced by the interaction between second - order dispersion, self - and quintic - phase modulations, so its aspect is different compared to that observed in [60]. In addition, the Akhmediev - Peregrine waves illustrated in Figure 2(c) are small and very narrow compared to that seen in [60] which are very large. This difference is due to the action of quintic - phase modulation $\alpha$ $=0.2 \times 10^{-25}$, which has deeply modified the waves field depicted in Figure 2(c). Moreover, this last remark is well confirmed by the dynamic of collective coordinates which exhibit significant internal modification in the system as illustrated in Figure 2(a). This behavior is different to that depicted in [60]. In fact, the action of quantic - phase modulation has modified the nature of internal excitation called modulation instability [38, 40]. This situation is confirmed by the dynamics of collective coordinates due to bare approximation (type II Ansatz function) which are limited in the interval of distance $0<Z<0.45 \times 10^{-24} \mathrm{~m}$ compared to those obtained in [60]. So, the type II Ansatz function produces collective coordinates which are not able to predict the behavior exhibited by the light pulse since they are limited in the space as depicted in Figure 2(a). But, type II ansatz function gives two additional coordinates $X 7$ and $X 8$ which produce supplemental information on frequency shift and chirp modifications. In addition, as depicted in Figure 2(a), the dynamics of collective coordinates coming from minimization adopt one broken point and linear behavior compared to that obtained in [60] which has exhibited several broken and random behavior. It is clearly appeared that quintic - phase modulation hardly modifies the system. We consider an increase of frequency from $\omega=0.25 \mathrm{rad} / \mathrm{s}$ to $\omega=$ $0.35 \mathrm{rad} / \mathrm{s}$ and Figure 2(e) is obtained. In fact, the Akhmediev - Peregrine waves field [84] depicted in Figure 2(c) is 
completely transformed into a single Peregrine wave illustrated in Figure 2(e). This single rogue event exhibits on the left part a generation of continual sidelobes similar to those often induced by third - order dispersion [57]. So, it appears that quintic - phase modulation has delated the part of field containing Akhmediev breathers which are replaced by a continual generation of sidelobes as depicted in Figure 2(e). If the frequency continues to increase from $\omega=0.35$ $\mathrm{rad} / \mathrm{s}$ to $\omega=0.78 \mathrm{rad} / \mathrm{s}$, the system regains in stability as depicted in Figure 2(b). However, this stability is not completely obtained since the three curves depicted in Figure 2(b) are not superimposed. One can suggest that the perturbation coming from quintic - phase modulation continues to act in the system. Hence, the stability of the light pulse is totally obtained for $\omega>0.78 \mathrm{rad} / \mathrm{s}$ as seen in Figures 2(d) and (e). In fact, the 2D and full numerical equations represent stable soliton light pulses which are depicted in Figures 2(d) and (e). In addition, the transmission line presents high perturbations at low frequencies and a stable propagation at high frequency. This behavior represents the main characteristic of the right - handed propagation.

\subsubsection{Generation of Special Structures}

We consider $C r=0$ and $\omega=1 \mathrm{rad} / \mathrm{s}$. The quintic - phase modulation increases from $\alpha=0.2 \times 10^{-25}$ to $\alpha=0.2 \times 10^{-21}$ and we obtain Figure 3. Compared to Figure 2(a), the dynamic of collective coordinates due to bare approximation are developed during all the length of propagation as depicted in Figure 3(a). However, they are maintained close to zero. Moreover, the dynamic of collective coordinates coming from minimization exhibit one group of broken points as depicted in Figure 2(a). In fact, the broken point appears at $\mathrm{Z}=0.2 \times 10^{-24} \mathrm{~m}$. After this distance, all the dynamic of collective coordinates adopt linear and constant values. This kind of modification suggests the birth of an extreme event as depicted in Figure 3. Thus, the residual field energy sustains this assertion since its value is maintained around 100 percent. This value suggests the activity of a strong internal agitation as seen in Figure 3(a). This high internal excitation leads to Figure 3(b). The exact field solution of the nonlinear Schrödinger equation is tried to maintain its Gaussian aspect. However, the high value of the dynamics of FWHM depicted in Figure 3(a), justifies the large structure presented in Figure 3 (b). The field reconstructed by collective coordinates due to type II Ansatz function exhibits the aspect of a small narrow rogue wave. This narrow wave is not superimposed to exact field, so those collective coordinates are not able to reconstruct the behavior of the exact field. The field reconstructed by collective coordinates originating from minimization is completely destroyed as shown in Figure 3 (b). The corresponding full numerical equation is illustrated in Figure 3(c) where a strange extreme event is represented. This extreme event is large at the low part and very narrow at the high part. So, its size gradually decreases from the low part to the high part as depicted in Figure 3(c). Moreover, this behavior is matched with that described by the corresponding exact field depicted in Figure 3(b).
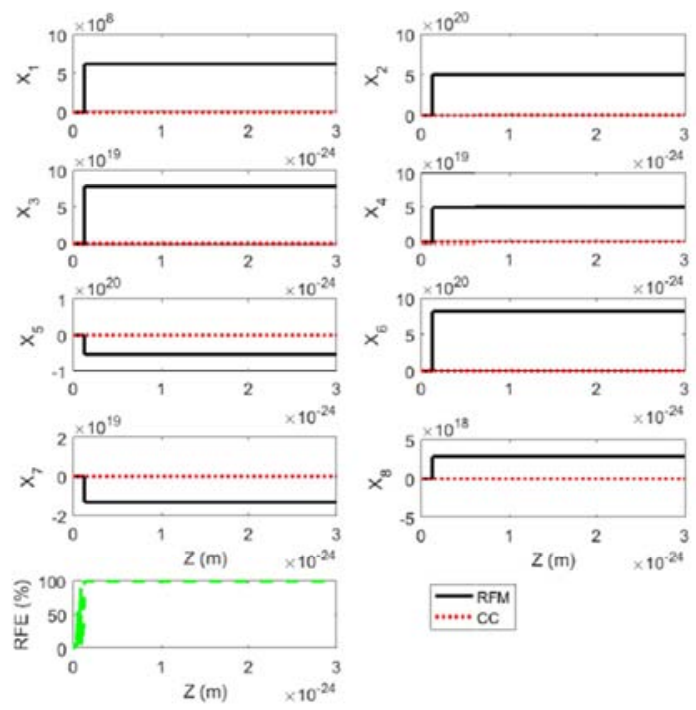

(a)

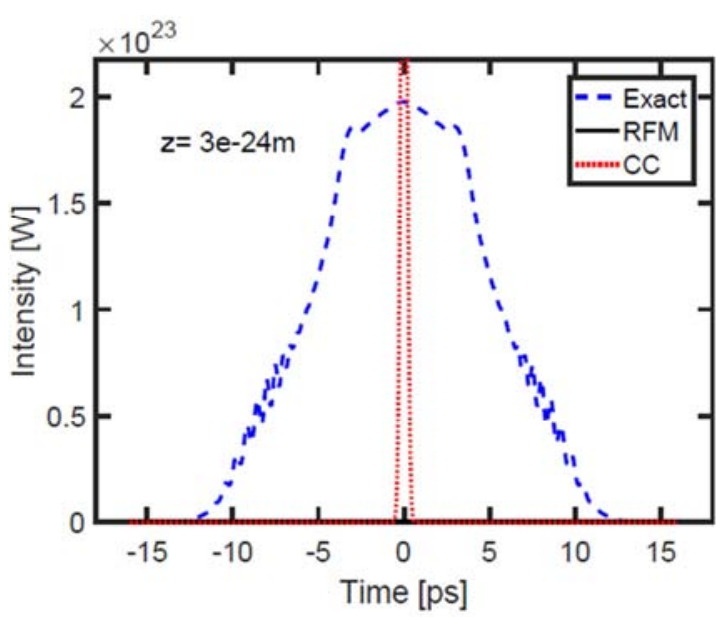

(b)

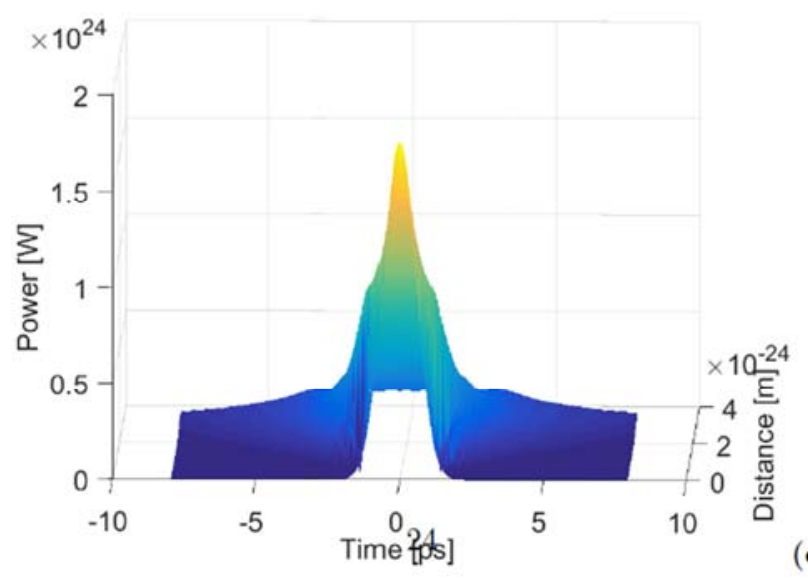

(c)

Figure 3. Generation of special structures at $Z=3 \times 10^{-24} \mathrm{~m}$ for $\mathrm{Cr}=0$ (Right handed behavior) when $\omega=1 \mathrm{rad} / \mathrm{s}$. Soliton light pulse subjected to quintic phase modulation $\alpha=0.2 \times 10^{-21}$ : (a) Dynamic of collective coordinates; (b) Intensity of fields; (c) Full numerical equation [ Frequency ranges considered: $0<\omega \leq 0.78 \$ \mathrm{rad} / \mathrm{s}$ and $\omega>0.78 \mathrm{rad} / \mathrm{s}]$.

\subsubsection{Multi- Wave Trains Generation and Tree Structure}

We consider the second case of right - handed behavior of the line such as $C r=0.3$ and $\omega=0.15 \mathrm{rad} / \mathrm{s}$. Then, quintic phase modulation continues to act such as $\alpha=0.2 \times 10^{-25}$ and 
we obtain Figure 4(a). The dynamic of collective coordinates due to bare approximation are limited in one interval of distance $0<Z<0.45 \times 10^{-24} \mathrm{~m}$ as seen in Figure 4(a). This behavior reveals that the system is hardly distorted by quintic - phase modulation and the type II Ansatz function cannot predict the behavior of the light pulse during all the distance of the propagation. However, the frequency shift and chirp correction factors give additional details on the internal excitation which strongly distorts the system as depicted in Figure 4(a). Moreover, the dynamics of amplitude and that of chirp correction factor present one group of broken points at
$Z=0.2 \times 10^{-24} \mathrm{~m}$. So, these two dynamic exhibit high values suggesting that the propagation will be done with the birth of extreme events associated with a strong modification of the chirp as shown in Figure 4(a). According to collective coordinates coming from minimization one group of broken points appears at $Z=0.1 \times 10^{-24} \mathrm{~m}$. After this distance, the dynamic of collective coordinates due to minimization adopt linear behavior as depicted in Figure 4(a). It appears that type II ansatz function produces collective coordinates unable to reconstruct those coming from minimization.
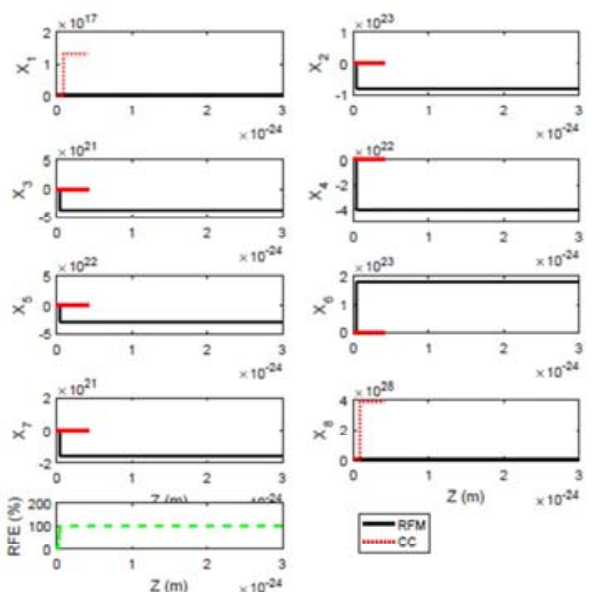

(a)
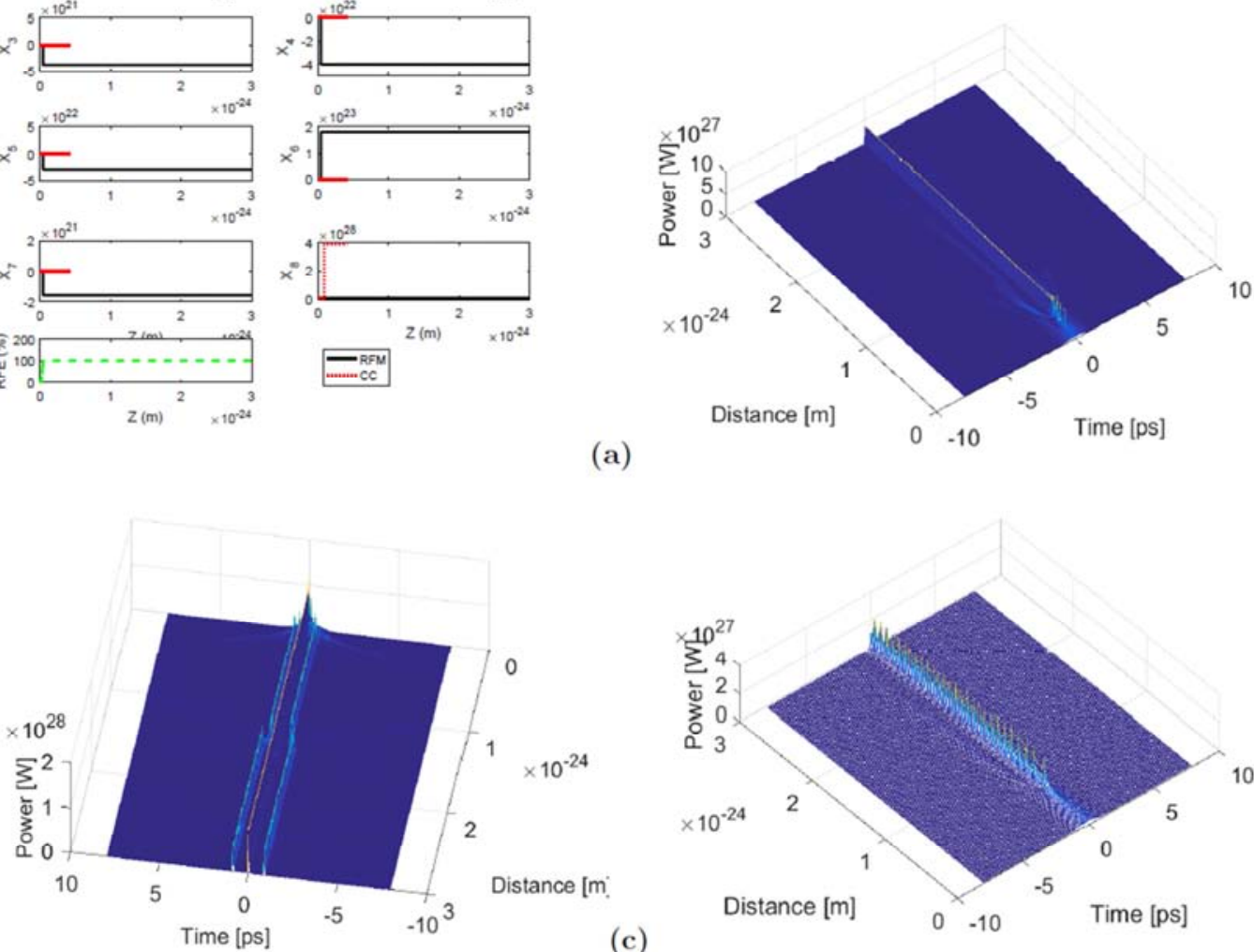

(c)

(d)
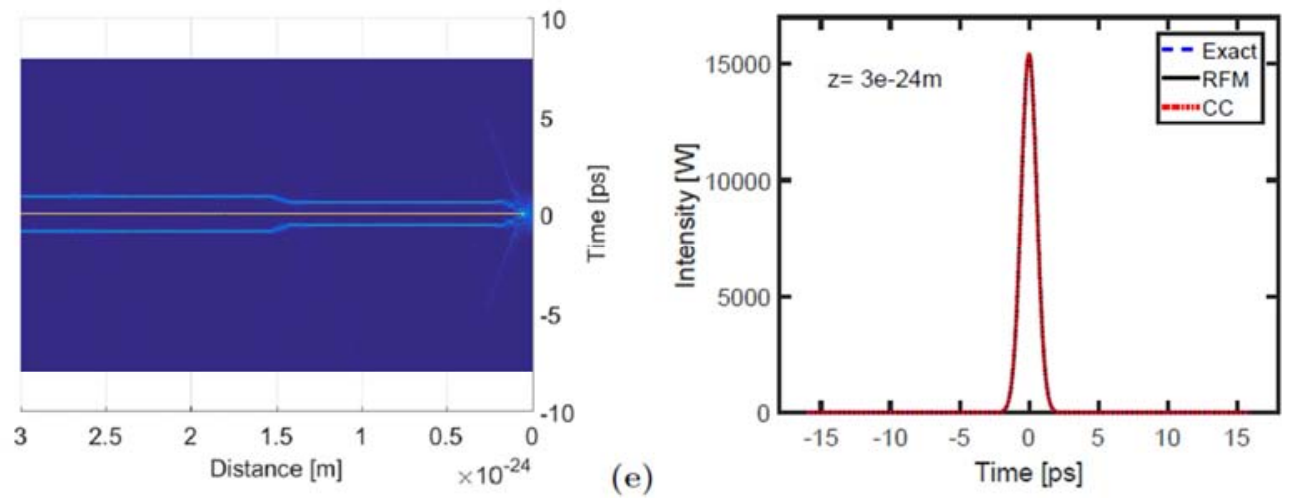

(b)

Figure 4. Generation of wall of waves, tree structure and Kuznetsov - Ma breathers at $Z=3 \times 10^{-24} \mathrm{~m}$ for $\mathrm{Cr}=0.3$ (Right - handed behavior). Soliton light pulse subjected to quintic - phase modulation $\alpha=0.2 \times 10^{-25}$ : (a) Dynamic of collective coordinates when $\omega=0.15 \mathrm{rad} / \mathrm{s}$; Full numerical equations: (b) $\omega=0.20 \mathrm{rad} / \mathrm{s}$, (c) $\omega=0.15 \mathrm{rad} / \mathrm{s}$ and (d) $\omega=0.25 \mathrm{rad} / \mathrm{s}$; (e) $2 D$ full numerical equation when $\omega=0.15 \mathrm{rad} / \mathrm{s}$; (f) Intensity of fields when $\omega=0.15 \mathrm{Mrad} / \mathrm{s}$ [ Frequency ranges considered: $0<\omega \leq 0.78 \mathrm{rad} / \mathrm{s}$ and $\omega>0.78 \mathrm{rad} / \mathrm{s}$. 
The dynamic of collective coordinates depicted in Figure 4 (a) represent the exact measure of the internal excitation leading to the extreme event illustrated in Figure 4(c). We observe the generation of three continual wave trains. These wave trains are very narrow and are similar to three walls. Moreover, the structure is composed by a main waves train at the middle of the system which is surrounded by two lateral wave trains as depicted in Figure 4(c). Previous investigations have shown that a wave train is created by modulation instability as seen in [33, 40, 59, 60]. However, it appears that the nature of modulation instability has changed in order to induce the generation of three wave trains as depicted in Figures 4(a) and (c). The main waves train at the middle corresponds to the brown straight line depicted in Figure 4(e) where the 2D full numerical equation is presented The two lateral wave trains correspond to the two blue curves. Each of these wave trains has undergone a deviation of sense at the distance $Z=1.5 \times 10^{-24} \mathrm{~m}$. This strange behavior suggests the modification of the modulation instability responsible to a such behavior as illustrated in Figures 4(a) and (e). At the beginning of the propagation, we observe some divergent waves similar to roots. So, the structure illustrated in Figure 4(e) is called tree structure [46]. The corresponding "roots" are called roots of propagation. This kind of structure corresponds to the signature of analytic nonlinear Schrödinger equation solutions in chaotic modulation instability as predicted $[46,47,49,50,83]$.

\subsubsection{Kuznetsov - Ma Wave Breathers}

We consider an increase of frequency from $\omega=0.15 \mathrm{rad} / \mathrm{s}$ to $\omega=0.20 \mathrm{rad} / \mathrm{s}$ and the quintic - phase modulation is maintained such as $\alpha=0.2 \times 10^{-25}$. The increase of frequency has induced the reduction of quintic - phase modulation strength of distortion. This situation has provoked the disappearance of the two lateral wave trains previously observed in Figures 4(b) and (e). This fact induces the generation of the single waves train depicted in Figure 4(b). Another increase of frequency from $\omega=0.20 \mathrm{rad} / \mathrm{s}$ to $\omega=$ $0.25 \mathrm{rad} / \mathrm{s}$ continues to strongly modify the modulation instability [33, 40, 59, 85], in order to provoke the generation of the Kuznetsov - Ma waves train [59] depicted in Figure 4 (f). This waves train generated by particular modulation instability has been well investigated in [59]. Besides, we consider a significant increase of frequency from $\omega=0.25$ $\mathrm{rad} / \mathrm{s}$ to $\omega=0.15 \mathrm{Mrad} / \mathrm{s}$ leading to the stable structure obtained in Figure 4(f). This structure is obtained by a suitable compensation process [46], between second - order dispersion, self - and quintic - phase modulations. The so called compensation process induced by an increase of frequency provokes the superimposition of the three curves depicted in Figure 4(f). This situation suggests the regain of the stability by the soliton light pulse. So, Figure 4(f) reveals the stability and the robustness of this wave called soliton light pulse [46]. It appears that Figure 4 suggests a high distorted environment at low frequencies, but a stable propagation at high frequencies. This behavior is same to that obtained in Figure 2. This is the traditional behavior of right handed propagation. So, the line has adopted the right handed behavior when $0<C r<0.69$.

\subsection{Left - Handed Behavior of Electrical Transmission Line}

The left - handed behavior of the line appears when $\mathrm{Cr}>$ 0.69 . Two cases of left - handed behavior are investigated such as $C r=1$ and $C r=10$.

\subsubsection{Stability of Light Pulse at Low Frequencies}

We consider the first case of left - handed behavior of the line such as $C r=1$ and we obtain Figure 5(a) for $\omega=0.15$ $\mathrm{rad} / \mathrm{s}$ and $\alpha=0.2 \times 10^{-25}$. The structure of the line has not changed but its behavior is modified. The dynamic of collective coordinates coming from bare approximation are reconstructed by those originating from minimization as depicted in Figure 5(a). This situation reveals the stability of the light pulse suggesting a perfect compensation process between second - order dispersion, self - and quantic - phase modulations [46]. Moreover, the precedent assertion is confirmed by residual field energy since its value is maintained around 0.05 percent suggesting a good reconstruction of the light pulse behavior by type II Ansatz function as seen in Figure 5(a). The stability depicted in Figure 5(a) is also confirmed in Figure 5(c) since the three curves are completely superimposed. This situation reveals the presence of a solid and stable wave called soliton light pulse as illustrated in Figure 5(c).

\subsubsection{Generation of Special Structures at High Frequencies}

We consider a significant increase of frequency from $\omega=0.15$ $\mathrm{rad} / \mathrm{s}$ to $\omega=25 \mathrm{Mrad} / \mathrm{s}$. The distance of propagation also increases from $Z=3 \times 10^{-24} \mathrm{~m}$ to $Z=10^{-6} \mathrm{~m}$ for $\alpha=0.2 \times 10^{-25}$ and Figure $5(\mathrm{~b})$ is obtained. The combination between the increase of frequency and the distance increases the quintic - phase modulation strength of distortion as seen in Figure 5(b). In fact, the exact field undergoes several distortions with a small extreme event at the middle surrounded on left and right by two continual generations of oscillations. In addition, similar investigations have been previously obtained in [60]. In fact, the structure presented in [60] is obtained by the action of cubic nonlinearity on second - order dispersion. The oscillating behavior seen in [60] is different compared to that depicted in Figure 5(b) since it has been obtained by the combination of second - order dispersion, self - and quintic - phase modulations. Another difference is that the structure in [60] has been studied by a Gaussian Ansatz function compared to that presented in Figure 5(b) which is presented by type II Ansatz function. Moreover, the field reconstructed by collective coordinates due to type II Ansatz function, is completely destroyed as seen in Figure 5(b).

However, the field reconstructed by collective coordinates coming from minimization is maintained its Gaussian behavior. Consequently, these two fields are not able to 
reconstruct the chaotic behavior exhibited by the exact field as seen in Figure 5(b). It has been demonstrated that Figure 5 (b) is associated to the generation of Akhmediev - Peregrine wave's field as shown in [60]. It appears that type II ansatz function cannot produce collective coordinates able to reconstruct the dynamics exhibited by the exact field [60]. Nonetheless, we consider a significant increase of quintic phase modulation from $\alpha=0.2 \times 10^{-25}$ to $\alpha=0.2 \times 10^{-2}$. Thereafter, we also consider a decrease of frequency from $\omega$ $=25 \mathrm{Mrad} / \mathrm{s}$ to $\omega=0.25 \mathrm{Mrad} / \mathrm{s}$. The combination of these two facts leads to the generation of the structure depicted in Figure 5(d). In addition, the exact field is transformed into a single and narrow extreme event as depicted in Figure 5(d). However, the field reconstructed by collective coordinates originating from minimization is practically reconstructed the behavior exhibited by the exact field as illustrated in Figure 5(d). Moreover, the field reconstructed by collective coordinates due to bare approximation is totally destroyed. The behavior exhibited in Figure 5(d) is similar to that often associated to Kuznetsov - Ma waves train as shown in [59]. It is clearly appeared that propagation is stable at low frequencies and unstable at high frequencies for the first case of left - handed propagation. This behavior is a traditional behavior of left - handed propagation as shown in [56].

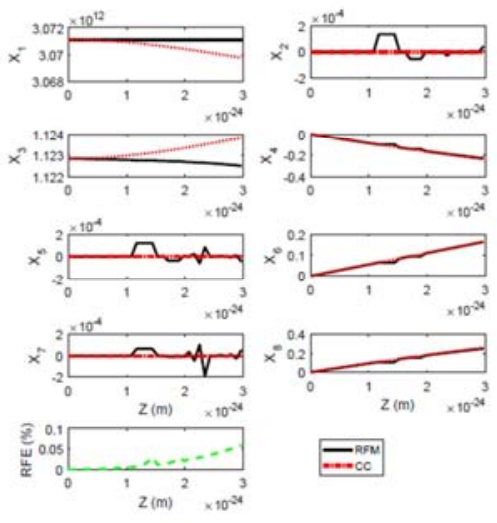

(a)

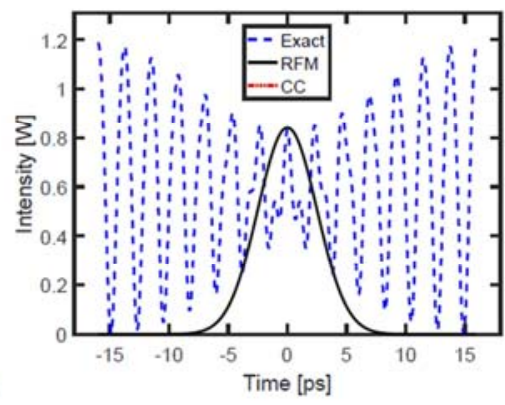

(b)
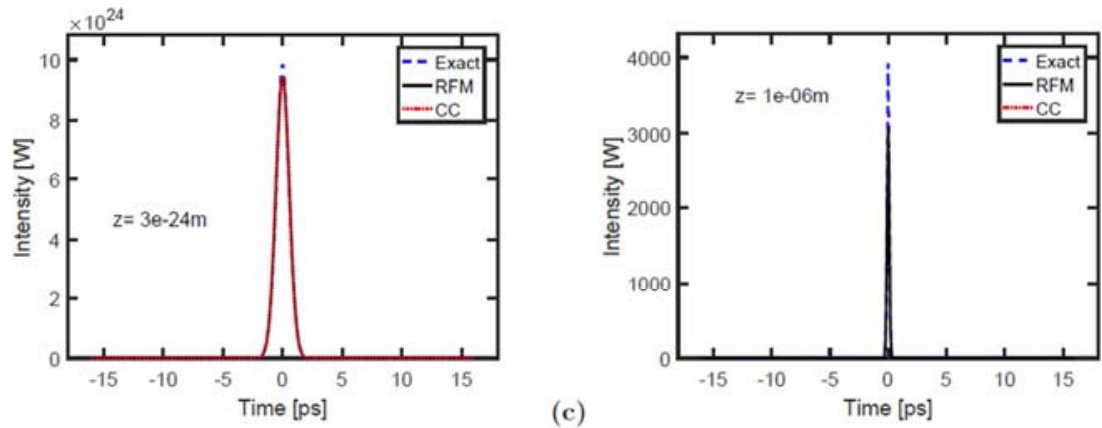

(d)

Figure 5. Generation of special structures for $\mathrm{Cr}=1$ (Left - handed behavior) at the distance $Z=3 \times 10^{-24} \mathrm{~m}$ (Left) and $Z=10^{-6} \mathrm{~m}$ (Right). (a) Dynamic of collective coordinates when $\omega=0.15 \mathrm{rad} / \mathrm{s}$; Intensity of fields: (b) $\omega=25 \mathrm{Mrad} / \mathrm{s}$, (c) $\omega=0.15 \mathrm{rad} / \mathrm{s}$ and (d) $\omega=0.25 \mathrm{Mrad} / \mathrm{s}$. Soliton light pulse subjected to quintic - phase modulation: (a), (b) and (c) obtained for $\alpha=0.2 \times 10^{-25}$. (d) obtained for $\alpha=0.2 \times 10^{-2}$ [ Frequency ranges considered: $0<\omega \leq 0.78$ rad/s and $\omega>0.78 \mathrm{rad} / \mathrm{s}]$.

\subsubsection{Generation of Triangular Rogue Waves at High Frequencies}

We consider the second case of left - handed behavior such as $C r=10$ for $\omega=25 \mathrm{Mrad} / \mathrm{s}$ and we obtain Figure 6(a). The dynamic of collective coordinates due to bare approximation are limited in the interval of distance $0<Z<0.2 \times 10^{-6} \mathrm{~m}$. One can suggests that the type II ansatz function cannot predict the behavior of the pulse during all the distance of propagation as seen in Figure 6(a). However, the dynamic of collective coordinates due to bare approximation undergo one group of broken points at $Z=0.1 \times 10^{-6} \mathrm{~m}$. The dynamics of phase and that of chirp correction factor exhibit high values. Then, type II Ansatz predicts propagation with high perturbations on the dynamic of phase and chirp correction factor. Considering the dynamic of collective coordinates originating from minimization, they present a different behavior compared to those due to bare approximation as seen in Figure 6(a). Further, the dynamic of temporal position, frequency shift and frequency shift correction factor undergo several distortions at the end of the propagation as seen in Figure 6(a). This behavior cannot be predicted by type II Ansatz function during the propagation. Moreover, the residual field energy is maintained around 100 percent suggesting the activity of high perturbation in the system. Consequently, this internal high disturbance will probably create an extreme event with small amplitude peaks as depicted in Figure 6(a) by the dynamics of amplitude. The dynamic of collective coordinates depicted in Figure 6(a) correspond to the exact measure of internal excitation leading to the generation of the extreme event seen in Figure 6 (b). Besides, the exact field corresponds to a periodical and 
distorted wave which cannot be reconstructed by the field due to minimization. This periodical and distorted wave cannot be reconstructed by the field coming from type II Ansatz function since it is destroyed as seen in Figure 6(b).
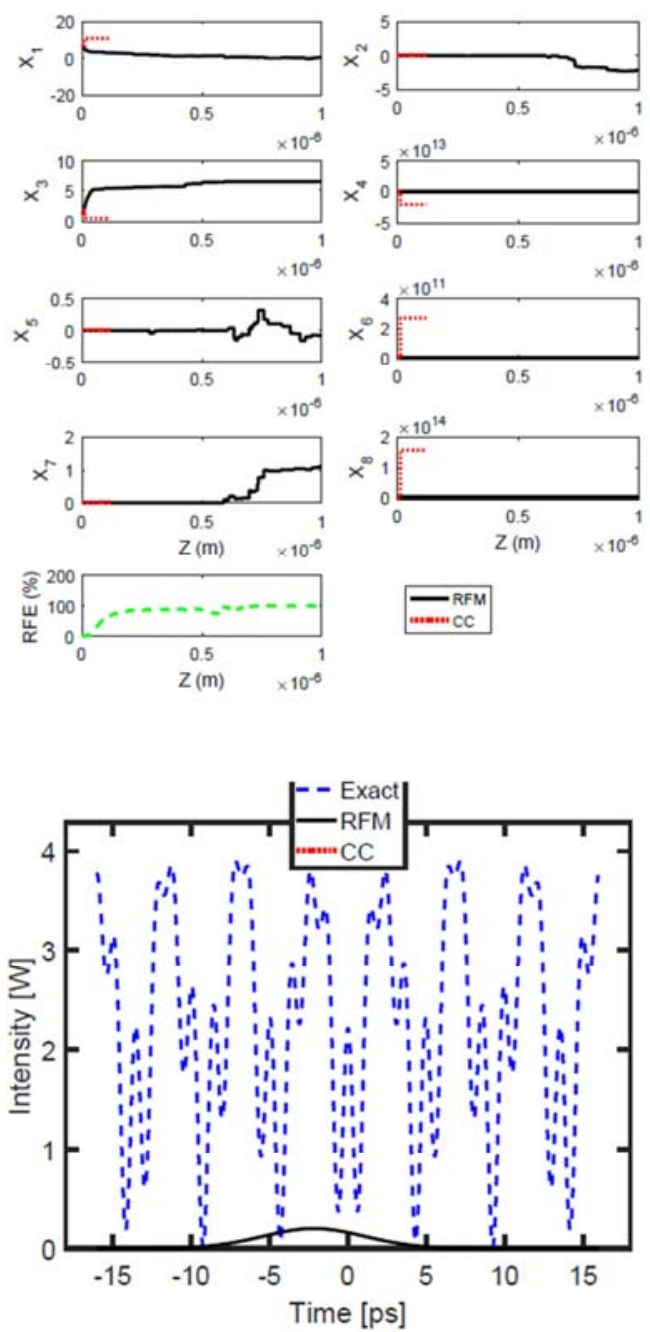

(a)

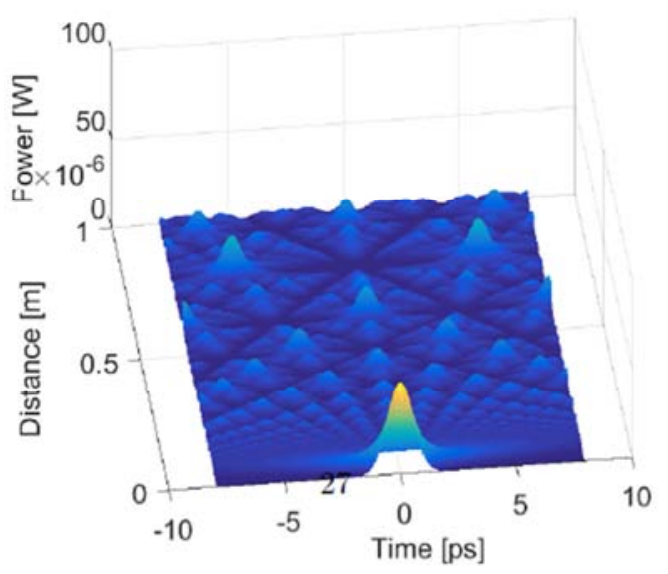

(b)

Figure 6. Generation of triangular rogue waves at $Z=10^{-6} \mathrm{~m}$ for $C_{r}=10$ (Left - handed behavior) when $\omega=25 \mathrm{Mrad} / \mathrm{s}$. Soliton light pulse subjected to quintic - phase modulation $\alpha=0.2 \times 10^{-25}$ : (a) Dynamic of collective coordinates; (b) Intensity of fields; (c) Full numerical equation [ Frequency ranges considered: $0<\omega \leq 0.78 \mathrm{rad} / \mathrm{s}$ and $\omega>0.78 \mathrm{rad} / \mathrm{s}]$.
The corresponding full numerical equation associated to the previous dynamic of collective coordinates and the periodical and distorted exact field, is illustrated in Figure 6(c). In fact, this structure is composed by several Peregrine waves which are positioned in order to build up many triangles [47, 86 - 89]. So, one triangle formed by these Peregrine waves is called triangular rogue waves as seen in Figure 6(c). Similar investigations have been made in [86] where Darboux transformation technique has been numerically applied. In fact, the authors have studied high order rational solutions of the nonlinear Schrödinger equation that appear spatiotemporally as triangular arrays of Peregrine solitons [86]. Moreover, they have obtained six Peregrine solitons which constitute quanta. Those Peregrine solitons have been separated and the distances between the adjacent peaks were the same. So, this structure has been called rogue wave cascade [86]. Another investigation using Darboux transformation, has also obtained similar results [88]. Further, triangular rogue waves have been also presented in the case of rational solutions of the Boussinesq equation [89]. This soliton equation has been solvable by inverse scattering [89]. Nevertheless, it is important to make a deep analysis of the parameter $\mathrm{Cr}$ which modifies the behavior of the line. In fact, we show that it fixes whenever the line can exhibit left - or right - handed behavior. But, a rigorous observation shows that a significant increase of this parameter in left - handed propagation can amplify the radiations in the system in order to build up the wave's field depicted in Figure 6(c). This strange behavior is similar to that observed in metamaterials when absorption regime is considered [46, 90, 91, 92, 93]. Absorption regime is a particular case of left - handed regime (negative index regime). Consequently, the next investigations will probably verify the real impact of $\mathrm{Cr}$ parameter when the different regimes [46], come into play.

\section{Conclusion}

In summary, we have shown that a particular line called chameleon transmission line can support the propagation of soliton light pulse subjected to quintic - phase modulation. The investigation has been done by collective coordinate's theory using an upgraded function called "type II Ansatz function" with eight coordinates compared to conventional Gaussian Ansatz with six coordinates. The strength of distortion of quintic - phase modulation has been studied when the line has adopted alternatively right - and left handed behaviors. The internal excitation due to quintic phase modulation has been measured by "type II Ansatz function" where the two additional coordinates have given supplemental details on frequency shift and chirp. According to conditions employed, the nature of this internal excitation has been modified in order to build up some extraordinary phenomena such as multi - wave trains, triangular rogue waves, the so - called "wall of waves", tree structure, Peregrine solitons, Akhmediev and Kuznetsov - Ma breathers. The most important point of this investigation is that special structure's generation is strongly influenced by the behavior 
of the line and the strength of distortion of quintic - phase modulation. Furthermore, many outlined points have a major physical interest. One, the determination of theoretical frequencies where the soliton safeguards its robustness. Hence, it is adaptable to data transmission at certain conditions for left -or right - handed propagation. Two, the internal and external conditions inducing the generation of particular structures. Three, additional details given by "type II Ansatz function" which improves the comprehension of rogue wave's mechanism of generation. The above mentioned results can improve the design of new telecommunication media.

\section{Acknowledgements}

The authors thank the anonymous reviewers, and the associate editor in charge of this manuscript. Special thanks to Professor Frédéric BIYA - MOTTO for its significant contribution.

\section{References}

[1] V. G. Veselago, The electrodynamics of substances with simultaneously negative values of $\varepsilon$ and $\mu$, Sov. Phys. Uspekhi (1968); 10: 509 - 514.

[2] B. Tütüncü, H. Torpi, B. Urul, A comparative study on different types of metamaterials for enhancement of microstrip patch antenna directivity at the Ku-band $(12 \mathrm{GHz})$, Turk J. Elec. Eng. and Comp. Sci. (2018); 26: 1171 - 1179.

[3] C. Caloz, H. Okabe, T. Iwai and T. Itoh, Transmission line approach of left-handed (LH) materials, USNC/URSI national radio science meeting, San Antonio, TX, June (2002); 1: 39.

[4] G. V. Eleftheriades, A. K. Iyer and P. C. Kremer, Planar negative refractive index media using periodically L-C loaded transmission lines, IEEE Trans Microwave Theory Tech. (2002); 50: 2702 - 2712 .

[5] A. B. Kozyrev and D. W. Vd. Weide, Nonlinear left-handed transmission line metamaterials, J. Phys. D (2008); 41:173001 -173010 .

[6] A. B. Kozyrev and D. W. van der Weide, Nonlinear wave propagation phenomena in left-handed transmission-line media, IEEE Trans Microwave Theory Tech. (2005); 53: 238 245.

[7] Gharakhili, F. G.; Shahabadi, M.; Hakkak, M. Bright and dark soliton generation in a left-handed nonlinear transmission line with series nonlinear capacitors, Progress In Electromagnetics Research, PIER (2009); 96: 237 - 249.

[8] Z. Wang, Y. Feng, B. Zhu, J. Zhao and T. Jiangn, Dark Schrödinger solitons and harmonic generation in left-handed nonlinear transmission line, J. Appl. Phys. (2010); 107: 094907.

[9] J. Ogasawara and K. Narahara, Experimental characterization of left-handed transmission line with regularly spaced Schottky varactors, IEICE Electron. Express. (2010); 7(9): $608-614$.

[10] L. Q. English, S. G. Wheeler, Y. Shen, G. P. Veldes, N.
Whitaker, P. G. Kevrekidis et al., Backwardwave propagation and discrete solitons in a left-handed electrical lattice, Phys. Lett. A (2011); 375: 1242.

[11] S. Abdoulkary, L. Q. English and A. Mohamadou, Envelope solitons in a left-handed nonlinear transmission line with Josephson junction, Chaos Solitons Fractals (2016); 85: 44 - 50.

[12] A. Lai, C. Caloz and T. Itoh, Composite right/ left-handed transmission line metamaterials, IEEE Microwave Mag. (2004); 5:34 - 50 .

[13] K. Narahara, T. Nakamichi, T. Suemitsu, T. Otsuji and E. Sano, Development of solitons in composite right- and left-handed transmission lines periodically loaded with Schottky varactors, J. Appl. Phys. (2007); 102: 024501.

[14] K. Narahara, Harmonic resonance in a composite right-handed and left-handed transmission line periodically loaded with Schottky varactors, Int. J. Circ. Theor. Appl. (2015). doi: 10.1002/cta. 2089.

[15] J. Ogasawara and K. Narahara, Short envelope pulse propagation in composite right-and left-handed transmission line with regularly spaced Schottky varactors, IEICE Electron. Express. (2009); 6:1576-1581.

[16] G. P. Veldes, J. Cuevas, P. G. Kevrekidis and D. J. Frantzeskakis, Quasidiscrete microwave solitons in a splitring-resonator-based left-handed coplanar waveguide, Phys. Rev. E (2011); 83: 046608.

[17] G. P. Veldes, J. Cuevas, P. G. Kevrekidis and D. J. Frantzeskakis, Coupled backward - and forwardpropagating solitons in a composite right-and left-handed trans- mission line, Phys. Rev. E (2013); 88: 013203.

[18] S. Abdoulkary, A. D. Aboubakar, M. Aboubakar, A. Mohamadou and L. Kavitha, Solitary wave solutions and modulational instability analysis of the nonlinear Schrödinger equation with higher-order nonlinear terms in the left-handed nonlinear transmission lines, Commun. Nonlinear Sci. Numer. Simulat. (2015); 22: 1288 - 1296.

[19] C. Kharif, E. Pelinovsky, and A. Slunyaev, Rogue Waves in the Ocean, (Springer, New York, 2009).

[20] E. Pelinovsky and C. Kharif (eds.), Extreme Ocean Waves, (Springer, New York, 2008).

[21] A. R. Osborne, Nonlinear Ocean Waves and the Inverse Scattering Transform, (Academic Press, Amsterdam, 2010).

[22] D. R. Solli, C. Ropers, P. Koonath, and B. Jalali, Optical rogue waves, Nature (2007); 450: 1054 - 1057.

[23] C. Lecaplain, Ph. Grelu, J. M. Soto-Crespo, and N. Akhmediev, Dissipative Rogue Waves Generated by Chaotic Pulse Bunching in a Mode-Locked Laser, Phys. Rev. Lett. (2012); 108: 233901.

[24] H. Bailung, S. K. Sharma and Y. Nakamura, Observation of Peregrine solitons in a multicomponent plasma with negative ions, Phys. Rev. Lett. (2011); 107: 255005.

[25] D. Peregrine, Water waves, nonlinear Schr"odinger equations and their solutions, Journal of the Australian Mathematical Society Series (1983); 25: $16-43$.

[26] Guo. B. L and Ling. L. M, Rogue Wave, Breathers and BrightDark-Rogue Solutions for the Coupled Schrödinger Equations, Chin. Phys. Lett. (2011); 28: 110202 - 4. 
[27] E. A. Kuznetsov, Solitons in a parametrically unstable plasma, Sov. Phys.-Dokl. (1977); 22: 575 - 577.

[28] Ya. C. Ma, The perturbed plane-wave solutions of the cubic Schrödinger equation, Stud. Appl. Math. (1979); 60: 43 - 58.

[29] N. Akhmediev, V. Eleonskii, N. Kulagin, Exact first - order solutions of the nonlinear Schrödinger equation, Theoretical and Mathematical Physics (1987); 72: 809 - 818.

[30] N. Akhmediev and A. Ankiewicz, Solitons: Non-linear Pulses and Beams (Chapman and Hall, 1997).

[31] N. Akhmediev, A. Ankiewicz, M. Taki, Waves that appear from nowhere and disappear without a trace, Phys. Lett. A (2009); 373: 675 - 678 .

[32] K. B. Dysthe and K. Trulsen, Note on breather type solutions of the NLS as models for freak-waves. Phys. Scripta T (1999); 82: $48-52$.

[33] V. Zakharov and L. Ostrovsky, Modulation instability: The beginning, Physica D. Nonlinear Phenom. (2009); 238: 540 548 .

[34] E. J. R. Kelleher, J. C. Travers, S. V. Popov and J. R. Taylor, Role of pump coherence in the evolution of continuous-wave supercontinuum generation initiated by modulation instability. J. Opt. Soc. Am. B (2012); 29: 502 - 512.

[35] J. M. Dudley, G. Genty, F. Dias, B. Kibler and N. Akhmediev, Modulation instability, Akhmediev breathers and continuous wave supercontinuum generation. Opt. Express (2009); 17, $21497-21508$

[36] A. I. Dyachenko and V. E. Zakharov, Modulation instability of Stokes wave — freak wave. JETP Lett. (2005); 81: 255 - 259.

[37] A. Chabchoub, N. Hoffmann, N. Akhmediev, Rogue wave observation in a water wave tank, Phys. Rev. Lett. (2011); 106 204502.

[38] I. Bespalov, V. I. Talanov, Filamentary structure of light beams in nonlinear liquids, JETP Lett. (1966); 3: 307 - 309.

[39] Y. Taniuti and H. Washimi, Self-trapping and instability of hydromagnetic waves along the magnetic field in a cold plasma, Phys. Rev. Lett. (1968); 21: 209.

[40] Benjamin, T. B.; Feir, J. E. J., The Disintegration of Wavetrains on Deep Water., Fluid Mech. (1967); 27: 417 - 430.

[41] P. Marqui'e, J. M. Bilbault and M. Remoissenet, Observation of nonlinear localized modes in an electrical lattice, Phys. Rev. E (1995); 51: 6127.

[42] V. V. Konotop and M. Salerno, Modulational instability in Bose- Einstein condensates in optical lattices, Phys. Rev. A (2002); 65: 021602(R).

[43] B. B. Baizakov, V. V. Konotop and M. Salerno, Regular spatial structures in arrays of Bose-Einstein condensates induced by modulational instability, J. Phys. B (2002); 35: 5105 - 5119.

[44] K. E. Strecker, G. B. Partridge, A. G. Truscott and R. G. Hulet, Formation and propagation of matter-wave soliton trains, Nature (2002); 417: 150 - 153.

[45] A. Hasegawa and Y. Kodama, Solitons in Optical Communications (Clarendon Press, Oxford, 1995).

[46] B. M. Ndi Nnanga, P. Y. Gouadjio Dontsop, B. G. Onana Essama,
Mohammed Shabat, David Yemele and Jacques Atangana, Treelike structures and Freak waves generation induced by quantic nonlinearity and cubic-Raman efect in a nonlinear metamaterial, Opt. Quan. Electron (2020); 52: 356 - 30.

[47] E. G. Charalampidis, J. Cuevas - Maraver, D. J. Frantzeskakis and P. G. Kevrekidis, Rogue waves in ultracold bosonic seas, Romanian Reports in Physics (2018); 70, 504 - 25.

[48] M. Bertola and A. Tovbis, Universality for the focusing nonlinear Schrödinger equation at the gradient catastrophe point rational breathers and poles of the Tritronqu'ee solution to Painlev'e I, Comm. Pure Appl. Math. (2013); 66, 678 - 752.

[49] C. Bayindir, Rogue waves of the Kundu-Eckhaus equation in a chaotic wave field, Phys. Rev. E (2016); 93: 062215.

[50] J. M. Dudley, F. Dias, M. Erkintalo and G. Genty, Instabilities, breathers and rogue waves in optics, Nature Photonics (2014); 8: $755-764$.

[51] David D. J. M. Snee and Yi-Ping Ma, Edge Solitons in a Nonlinear Mechanical Topological Insulator, Nonlinear Sciences, (2018) doi: arXiv:1805.03157v1.

[52] B. Liu, L. Li, B. A. Malomed, Stability band structure for periodic states in periodic potentials, Applied Numerical Mathematics, (2018); doi:arXiv:1805.07234v1.

[53] J. Tan, Z. H. Deng, T. Wu, B. Tang, Propagation and interaction of magnetic solitons in a ferromagnetic thin film with the interfacial Dzyaloshinskii - Moriya interaction, Journal of Magnetism and Magnetic Materials. (2019); 475: 445 - 452.

[54] C. Liu, R. E. C. van der Wel, N. Rotenberg, L. Kuipers, T. F. Krauss, A. Di Falco and A. Fratalocchi, Triggering extreme events at the nanoscale in photonic seas, Nature Physics, (2015); 11: $358-363$.

[55] B. G. Onana Essama, J. Atangana, B. Mokhtari, N. Cherkaoui Eddeqaqi and T. C. Kofane, Theoretical model for electromagnetic wave propagation in negative index material induced by cubic-quintic nonlinearities and third-order dispersion effects, Opt. Quant. Electron. (2013); 46: 911 - 924.

[56] B. G. Onana Essama, J. Atangana, F. Biya Motto, B. Mokhtari, N. Cherkaoui Eddeqaqi and Timoleon C. Kofane, Rogue waves generation in a left-handed nonlinear transmission line with series varactor diodes, J. Mod. Opt. (2014); 61: 1002 1008 .

[57] B. G. Onana Essama, J. Atangana, F. Biya Motto, B. Mokhtari, N. Cherkaoui Eddeqaqi and Timoleon C. Kofane, Optical rogue waves generation in a nonlinear metamaterial, Opt. Commun. (2014); 331: 334 - 347.

[58] B. G. Onana Essama, J. Atangana, F. Biya Motto, B. Mokhtari, N. Cherkaoui Eddeqaqi and Timoleon C. Kofane, Rogue wave trains generation in a metamaterial induced by cubic-quinticnonlinearities and second-order dispersion, Phys. Rev. E (2014); 90: 032911 - 12.

[59] J. Atangana, B. G. Onana Essama, F. Biya Motto, B. Mokhtari, N. Cherkaoui Eddeqaqi and Timoleon C. Kofane, Kuznetsov Ma waves train generation in a left-handed material, J. Mod. Opt. (2014); 62: 392 - 402.

[60] P. Y. Gouadjio Dontsop, B. G. Onana Essama, J. M. Dongo, M Mbou Dedzo, J. Atangana, D. Yemele, T. C. Kofane, Akhmediev-Peregrine rogue waves generation in a composite right/left-handed transmission line, Opt. Quant. Electron. (2016); 48: 59 - 77. 
[61] J. Atangana, B. M. Ndi Nnanga, B. G. Onana Essama, B. Mokthari, N. Cherkaoui Eddeqaqi, T. C. Kofane, Efficient method of calculation of Raman soliton self - frequency shift in nonlinear optical media, Opt. Commun. (2015); 339: 194 - 208.

[62] K. Fukushima, M. Wadati and Y. Narahara, Envelope soliton in a new nonlinear transmission line, J. Phys. Soc. Jpn. (1980); 49: 1593 - 1597.

[63] A. B. Togueu Motcheyo, J. D. Tchinang Tchameu, S. I. Fewo, C. Tchawoua, T. C. Kofane, Chameleon's behavior of modulable nonlinear electrical transmission line, Commun. Nonlinear Sci. Numer. Simulat. (2017); 53: 22 - 30.

[64] V. Shrira and V. Geogjaev, What makes the Peregrine soliton so special as a prototype of freak waves?, Journal of Engineering Mathematics (2010); 67: $11-22$.

[65] B. Kibler, J. Fatome, C. Finot, G. Millot, F. Dias, G. Genty, N. Akhmediev, J. Dudley, The Peregrine soliton in nonlinear fibre optics, Nature Physics, (2010); 6: 790 - 795.

[66] N. Akhmediev, V. Eleonskii, N. Kulagin, Generation of periodic trains of picosecond pulses in an optical fiber: exact solutions, Soviet Physics JETP (1985); 62: 894 - 899.

[67] N. Akhmediev, V. Korneev, Modulation instability and periodic solutions of the nonlinear Schrödinger equation, Theoretical and Mathematical Physics (1986); 69: 1089 1093.

[68] M. Onorato, S. Residori, U. Bortolozzo, A. Montina and F. T. Arecchi, Rogue waves and their generating mechanisms in different physical contexts, Phys. Rep. (2013); 528: 47 - 89.

[69] P. Tchofo Dinda, K. Nakkeeran and A. Labruyere, Suppression of soliton self-frequency shift by upshifted filtering, Opt. Lett. (2002); 27: $382-384$.

[70] P. Tchofo Dinda, A. B. Moubissi and K. Nakkeeran, A collective variable approach for dispersion managed solitons, J. Phys. A: Math. Gen. (2001); 34: 103 - 110.

[71] S. Fewo, H. Moussambi and T. C. Kofane, Analysis of nonparaxial solitons using collective variable approach, Phys. Scr. (2011); 84: 035002.

[72] S. Shwetanshumala and A. Biswas, Femtosecond pulse propagaton in optical fibers under higher order effects: A collective variable approach, J. Theor. Phys. (2008); 47: 1699 -1708 .

[73] Shwetanshumala, Temporal solitons in nonlinear media modeled by modified complex Ginzburg Landau Equation under collective variable approach S. J. Theor. Phys. (2009); 48: 1122 - 1131.

[74] R. Hirota and K. Suzuki, Studies on lattice solitons by using electrical networks, J. Phys. Soc. Jpn. (1970); 28: 1366 - 1367.

[75] R. Hirota and K. Suzuki, Theoretical and experimental studies of lattice solitons in nonlinear lumpednetworks, Proc. IEEE (1973); 61: 1483 - 1491 .

[76] G. P. Agrawal, Nonlinear Fiber Optics (Academic Press, San Diego, 2nd ed. 1995).

[77] J. Atangana, B. G. Onana Essama, B. Mokhtari and T. C. Kofane, Cubic-quintic saturable nonlinearity effects on a light pulse strongly distorted by the fourth-order dispersion. J. Mod. Opt. (2013); 60: 292 - 300.
[78] B. B. Baizakov, A. Bouketir, S. M. Al-Marzoug, H. Bahlouli, Effect of quintic nonlinearity on modulation instability in coupled nonlinear Schrödinger systems, Nonlinear Sciences, (2018); DOI:10.1016/j.ijleo.2018.11.092.

[79] M. Lobet, M. Lard, M. Sarrazin, O. Deparis and L. Henrard, Plasmon hybridization in pyramidal metamaterials: a route towards ultra-broadband absorption, Optics Express (2014); 22: $12678-12690$

[80] R. A. Van Gorder, Andrew L. Krause, and James A. Kwiecinski, Amplitude death criteria for coupled complex Ginzburg-Landau systems, Nonlinear Sciences, (2019); doi: arXiv:1803.02147.

[81] A. D. Boardman, A. Alberucci, G. Assanto, V. V. Grimalsky, B. Kibler, J. McNiff, I. S. Nefedov, Y. G. Rapoport and C. A. Valagiannopoulos, Waves in hyperbolic and double negative metamaterials including rogues and solitons, Nanotechnology (2017); 28: 444001.

[82] N. Akhmediev et al., Roadmap on optical rogue waves and extreme events, J. Opt. (2016); 18: 063001 - 38.

[83] J. M. Soto-Crespo, P. Grelu and N. Akhmediev, Dissipative rogue waves: Extreme pulses generated by passively modelocked lasers, Phys. Rev. E (2011); 84: 016604.

[84] Nikolay K. Vitanov, Amin Chabchoub, Norbert Hoffmann, Deep-Water Waves: On The Nonlinear Schrödinger Equation And Its Solutions, Journal of Theoretical and Applied Mechanics, (2013); 43: 43 - 54.

[85] Zakharov, V., Stability of Periodic Waves of Finite Amplitude on the Surface of a Deep Fluid, J. Appl. Mech. Tech. Phys. (1968); 9: $190-194$.

[86] D. J. Kedziora, A. Ankiewicz and N. Akhmediev, Triangular rogue wave cascades, Phys. Rev. E (2012); 86: 056602 - 9.

[87] R. H. J. Grimshaw and A. Tovbis, Rogue waves: Analytical predictions, Proc. Roy. Soc. A Math. Phys. Eng. Sci. (2013); 469: 2157 - 12.

[88] L. Wei, Q. De-Qin and H. Jing-Song., Localized Properties of Rogue Wave for a Higher-Order Nonlinear Schrödinger Equation, Commun. Theor. Phys. (2015); 63: 525 - 534.

[89] A. Ankiewicz, A. P. Bassom, P. A. Clarkson and E. Dowie, Conservation laws and integral relations for the Boussinesq equation, (2017);doi: arXiv:1611.09505v3 [nlin.SI].

[90] Alexander K. Popov and Vladimir M. Shalaev, Compensating losses in negative-index metamaterials by optical parametric amplification. Opt. Lett. (2006); 31: 2169 - 2171.

[91] N. M. Litchinitser and V. M. Shalaev, Loss as a route to transparency, Nat. Photonics, (2009); 3: 75 - 79.

[92] B. G. Onana Essama, S. Ndjakomo Essiane, F. Biya-Motto, B. M. N. Nnanga, M. Shabat, and J. Atangana: "Dynamical Evolution of Sasa-Satsuma Rogue Waves, Breather Solutions, and New Special Wave Phenomena in a Nonlinear Metamaterial". Phys. Stat. Sol. B. (2020); 2000316 - 24. DOI: $10.1002 /$ pssb.202000316

[93] B. M. N. Nnanga, P. Y. G. Dontsop, B. G. Onana Essama, M. Shabat, D. Yemele, J. Atangana: "Tree-like structures and Freak waves generation induced by quintic-nonlinearity and cubic-Raman effect in a nonlinear metamaterial”. Opt. Quant. Electron. (2020); 52: $356-30$. 